\title{
Global Existence of Classical Solutions to a Cancer Invasion Model
}

\author{
Khadijeh Baghaei $^{{ }^{*}}$, Mohammad Bagher Ghaemi ${ }^{1}$, Mahmoud Hesaaraki ${ }^{2}$ \\ ${ }^{1}$ Department of Mathematics, Iran University of Science and Technology, Tehran, Iran \\ ${ }^{2}$ Department of mathematics, Sharif University of Technology, Tehran, Iran \\ Email: "khbaghaei@iust.ac.ir
}

Received January 25, 2012; revised March 8, 2012; accepted March 15, 2012

\begin{abstract}
This paper deals with a chemotaxis-haptotaxis model of cancer invasion of tissue. The model consists of three reactiondiffusion-taxis partial differential equations describing interactions between cancer cells, matrix degrading enzymes, and the host tissue. The equation for cell density includes two bounded nonlinear density-dependent chemotactic and haptotactic sensitivity functions. In the absence of logistic damping, we prove the global existence of a unique classical solution to this model by some delicate a priori estimate techniques.
\end{abstract}

Keywords: Cancer Invasion Model; Chemotaxis; Haptotaxis; Global Existence

\section{Introduction}

Cancer invasion is associated with the degradation of the extra cellular matrix $(\mathrm{ECM})$, which is degraded by matrix degrading enzymes (MDEs) secreted by tumor cells. The degradation creates spatial gradients which direct the migration of invasive cells either via chemotaxis (cellular locomotion directed in response to a concentration gradient of the diffusible MDE) or via haptotaxis (cellular locomotion directed in response to a concentration gradient of adhesive molecules along the ECM). Chaplain and Lolas [1] proposed a PDE model of cancer invasion of tissue, which considers the competition between the following several biological mechanisms: random diffusion, chemotaxis, haptotaxis and logistic growth.

Actually, cancer invasion is a very complex process which involves many various biological mechanisms. In fact, a variety of mathematical models have been developed for various aspects of cancer invasion, and various attempts to give more biologically relevant models have been made by different people (see [2], for instance). Gatenby and Gawlinski [3] useda reaction-diffusion population competition model to study how the tumor invades the surrounding normal tissue or ECM. They suggested that tumor cells createan acidic environment that is toxic to normal tissue, and the high acidity gives rise to the death of the normal tissue, which provides space for tumor cells to proliferate and invade into the surrounding tissue. In contrast to the acid-invasion mechanism, Perumpanani and Byrne [4] found that the ECM heteroge-

${ }^{*}$ Corresponding author. neity affects suchinvasion. They proposed a model under the assumptions that the ECM is degraded by proteases. The proliferation of tumor cells and the remodeling of the ECM are taken into account in the Chaplain and Lolas model. Recently, Gerisch and Chaplain [5] developed a novel non-local model which incorporates cellcell adhesion and cell matrix adhesion, playing important roles in the tumor invasion process.

Very recently, Szymańka et al. [6] proposed a nonlocal model which focuses on the role of non-local kinetic terms modeling competition for space and degradation; Szymańka et al. [7] also discussed the influence of heat shock proteins on cancer invasion of tissue. The analytical results on various models of cancer invasion are mathematically interesting. Walker and Webb [8] proved the global existence solutions to the Chaplain and Anderson's model [9]. Walker [10] also established the global existence of solutions to an age and spatiallystructured haptotaxis model, which can be regarded as an extension of the Chaplain and Anderson's model [9]. Marciniak-Czochra and Ptashnyk recently [11] proved the uniform boundedness of solutions to the haptotaxis model [9]. Szymańka et al. [6] proved the global existence of solutions to their non-local model.

Very recently, by refining their previous techniques developed in [12]. Litcanu and Morales-Rodrigo [13] studied the asymptotic behavior of solutions to Perumpanani and Byrne's model [4]. Paper [13], to our knowledge, is the first attempt to analytically discuss the asymptotic behavior of solutions for cancer invasion models. We should note that the cancer invasion models in $[4-7,9,14]$ 
are haptotaxis only models. However, Chaplain and Lolas' model [1] is a parabolic-ODE-parabolic-chemotaxis-haptotaxis system. The global existence and uniqueness of classical solutions to this model has been proved for $\mu \geq 0$ (where $\mu$ is the growth rate of cancer cells) in one space dimension (see [15]), for $\mu>0$ in two space dimensions (see [16]) and for large $\mu$ in three space dimensions (see [15]).We should note that the global existence is still open for small $\mu>0$ in three space dimensions for the parabolic-ODE-parabolic chemotaxishaptotaxis system and the parabolic-ODE-elliptic chemotaxis-haptotaxis system.

Recently, in addition to global existence and uniqueness, the uniform-in-time boundedness of solutions to a simplified parabolic-ODE-elliptic-chemotaxis-haptotaxis system has been proved for $\mu>0$ in two space dimensions and for large $\mu$ in three space dimensions (see [17]).

This paper tries to analytically study a mathematical model of cancer invasion with $\mu=0$. When $\mu=0$, the solution Chaplain and Lolas' model can blow up in finite time (see Section 6, [15]). However, it is obvious that the blow-up of cancer cell density in finite time is biologically irrelevant. Hence, we need to deal with the following problem: how to reasonably modify the Chaplain and Lolas' model [1] to obtain the global existence, which is the cancer of the present paper.

This paper extends Chaplain and Lolas' model to a parabolic-parabolic-parabolic chemotaxis-haptotaxis system, and we study the global existence and boundedness of solutions to this model. This paper organized as follows: Section 2 describes the model. Section 3 proves the local existence and uniqueness of solutions. Section 4 establishes some a priori estimates and proves the global existence.

\section{Mathematical Model}

The mathematical model of cancer invasion is involved in the following three physical variables: cancer cell density $c(x, t)$, ECM density $v(x, t)$ and MDE concentration $u(x, t)$.

The equations describing the dynamics of each variable read as follows:

$$
\begin{aligned}
\frac{\partial c}{\partial t} & =\underbrace{D_{c} \Delta c}_{\text {random motion }}-\underbrace{\nabla \cdot\left(\chi V_{1}(c) \nabla u\right)}_{\text {randommotion }} \\
& -\underbrace{\nabla \cdot\left(\xi V_{2}(c) \nabla v\right)}_{\text {haptotaxis }}, \\
\frac{\partial v}{\partial t} & =\underbrace{D_{v} \Delta v}_{\text {random motion }}-\underbrace{F(u) v}_{\text {proteolysis }} \\
\frac{\partial u}{\partial t} & =\underbrace{D_{u} \Delta u}_{\text {diffusion }}+\underbrace{\alpha c}_{\text {production }}-\underbrace{\beta u}_{\text {decay }},
\end{aligned}
$$

where $D_{c}, D_{v}, D_{u}, \chi, \xi, \alpha$ and $\beta$ are assumed to be positive constants and $V_{1}(c)$ and $V_{2}(c)$ are the density-dependent chemotactic and haptotactic sensitivity functions, respectively.

In Equation (1), the migration of cancer cells is assumed to be governed by random motion, chemotaxis and haptotaxis. In Equation (2) is assumed that ECM has random motion and its degradation by MDEs upon contact; for simplicity, we assume that no remodeling of the ECM takes place, as done in $[15,18]$. Since random motion ECM is so small hence we assume that $D_{v}$ is small positive constant. In Equation (3), the MDE concentration is assumed to be influenced by diffusion, production and decay; specifically, MDE is produced by cancer cells, diffuses throughout ECM, and undergoes decay through simple degradation. We shall consider the system (1)-(3) in a bounded domain $\Omega$ in $\mathbb{R}^{d}(\mathrm{~d}=2$ or 3$)$.

For any $0<T<\infty$ we set

$$
\begin{gathered}
\Omega_{T}=\Omega \times\{0<t<T\}, \\
\partial \Omega_{T}=\partial \Omega \times\{0<t<T\} .
\end{gathered}
$$

To close the system of equations, we need to impose boundary and initial conditions.

\section{Boundary conditions:}

The boundary conditions are represented by the following equalities:

$$
\frac{\partial c}{\partial n}=\frac{\partial u}{\partial n}=\frac{\partial v}{\partial n}=0 \text { on } \partial \Omega_{T},
$$

where $n$ is the our ward normal vector to $\partial \Omega$.

Initial conditions: We prescribe the initial data

$$
\begin{aligned}
& c(x, 0)=c_{0}(x), v(x, 0)=v_{0}(x), \\
& u(x, 0)=u_{0}(x), x \in \Omega
\end{aligned}
$$

Throughout this paper we will assume that

$$
\begin{gathered}
V_{i}(c) \in C^{1}([0,+\infty)), V_{i}(c) \geq 0, V_{i}(0)=0, \\
V_{i}^{\prime}(c) \text { is Lipschitz continuous, }
\end{gathered}
$$

where $i=1,2$ and

$$
F(u) \in C^{1}([0,+\infty)) \text { and } F(u) \geq 0 .
$$

In Chaplain and Lolas' original model [1], it is assumed that $V_{1}(c)=\chi c, V_{2}(c)=\xi c$ and $F(u)=\gamma u$ (where $\chi, \xi$ and $\gamma$ are some positive constants). For this choice of $V_{1}(c), V_{2}(c)$ and $F(u)$, although the assumptions (6)(8) are satisfied but we would like to slightly modify the choice of $V_{1}(c), V_{2}(c)$ and $F(u)$ such that the modified model has a unique global solution. To this end, in addition to the assumptions (6)-(8), we will assume that

$$
\begin{gathered}
V_{1}(c) \text { and } V_{2}(c) \text { are bounded for any } c \geq 0, \\
F(u) \text { is bounded for any } u \geq 0 .
\end{gathered}
$$


For example, we may take $V_{1}(c)=\frac{\chi c}{1+\epsilon_{1} c}$,

$V_{2}(c)=\frac{\xi_{C}}{1+\epsilon_{2} c} \quad$ and $\quad F(u)=\frac{\delta u}{1+\epsilon_{3} u} \quad\left(\epsilon_{1}, \epsilon_{2}\right.$ and $\epsilon_{3}$ are small positive constants ). Clearly $V_{1}(c) \rightarrow \chi c$ as $\epsilon_{1} \rightarrow 0, V_{2}(c) \rightarrow \xi c$ as $\epsilon_{2} \rightarrow 0$ and $F(u) \rightarrow \delta u$ as $\epsilon_{3} \rightarrow 0$. For this choiceof $V_{1}(c), V_{2}(c)$, and $F(u)$, the assumptions (6)-(10) are satisfied. Another choice of $V_{1}(c)$ and $V_{2}(c)$ satisfying (9) is that $V_{1}(c) \equiv 0$ and $V_{2}(c) \equiv 0$ for $c \geq c_{m}$, which has a clear biologically relevant interpretation: the cancer cells stop to accumulate at a given point of the tumor tissue after their density attains a maximal density $c_{m}$. A similar assumption for a prey taxis sensitivity function was made in [19].

In next section we will prove the local existence and uniqueness of a solution for the system (1)-(5) by a fixed point argument.

\section{Local Existence and Uniqueness}

Throughout this paper we assume that

$$
\begin{aligned}
& c_{0}(x) \geq 0, v_{0}(x) \geq 0, u_{0}(x) \geq 0, \\
& \partial \Omega \in C^{2+\sigma}, 0<\sigma<1, \\
& c_{0}(x), v_{0}(x), u_{0}(x) \in C^{\sigma+2}(\bar{\Omega}), \\
& \frac{\partial c_{0}(x)}{\partial n}=\frac{\partial v_{0}(x)}{\partial n}=\frac{\partial u_{0}(x)}{\partial n}=0 \text { on } \partial \Omega .
\end{aligned}
$$

For brevity we set

$$
U=(c, v, u)
$$

For notations' convenience, in what follows we denote various constants which are independent of $T$ by $A_{0}$, whereas we denote various constants which depend on $\mathrm{T}$ by $A$. The constants $A_{0}$ and $A$ may be different from line to line.

In the following, under the assumptions (6)-(8) and (11), we shall prove that the system (1)-(5) has a unique local (in time) smooth solution.

Theorem 3.1. Under the assumptions (6)-(8), there existsa unique solution $U \in C^{2+\sigma, 1+\sigma / 2}\left(\Omega_{T}\right)$ of the system (1)-(5) for some small $T>0$ which depends on $\|U(., 0)\|_{C^{2+\sigma}(\Omega)}$.

Proof. We shall prove the local existence by a fixed point argument. We introduce the Banach space $X$ of the vector function $U$ (defined in (12)) with norm

$$
\|U\|=\|U\|_{C^{1+\sigma, \sigma / 2}\left(\Omega_{T}\right)}(0<T<1)
$$

and a subset

$$
X_{M}=\{U \in X:\|U\| \leq M\},
$$

where

$$
\begin{aligned}
M: & =\left\|c_{0}(x)\right\|_{C^{2+\sigma}(\Omega)}+\left\|v_{0}(x)\right\|_{C^{2+\sigma}(\Omega)} \\
& +\left\|u_{0}\right\|_{C^{2+\sigma}(\Omega)}+1 \\
= & \|U(., 0)\|_{C^{2+\sigma}(\Omega)}+1
\end{aligned}
$$

Given any $U \in X_{M}$, we define a corresponding.

Function $\bar{U}=F U$ by $\bar{U}=(\bar{c}, \bar{v}, \bar{u})$, where $\bar{U}$ satisfies the equations

$$
\begin{gathered}
\frac{\partial \bar{v}}{\partial t}-D_{v} \Delta \bar{v}+F(u) \bar{v}=0 \text { in } \Omega_{T}, \\
\frac{\partial \bar{v}}{\partial n}=0 \text { on } \partial \Omega_{T}, \\
\bar{v}(x, 0)=v_{0}(x) \text { in } \Omega, \\
\frac{\partial \bar{u}}{\partial t}-D_{u} \Delta \bar{u}+\beta \bar{u}=\alpha c \text { in } \Omega_{T}, \\
\frac{\partial \bar{u}}{\partial n}=0 \text { on } \partial \Omega_{T}, \\
\bar{u}(x, 0)=u_{0}(x) \text { in } \Omega, \\
\frac{\partial \bar{c}}{\partial t}-D_{c} \Delta \bar{c}=h_{1}(c, \bar{u}, \bar{v}) \text { in } \Omega_{T}, \\
\frac{\partial \bar{c}}{\partial n}=0 \text { on } \partial \Omega_{T}, \\
\bar{c}(x, 0)=c_{0}(x) \text { in } \Omega,
\end{gathered}
$$

where

$$
\begin{aligned}
h_{1}(c, \bar{u}, \bar{v})= & -\nabla \cdot\left(\chi V_{1}(c) \nabla \bar{u}\right) \\
& -\nabla \cdot\left(\xi V_{2}(c) \nabla \bar{v}\right) \\
= & -\chi V_{1}(c) \Delta \bar{u}-\xi V_{2}(c) \Delta \bar{v} \\
& -\left(\chi V_{1}^{\prime}(c) \nabla \bar{u}+\xi V_{2}^{\prime}(c) \nabla \bar{v}\right) \cdot \nabla c
\end{aligned}
$$

We first consider the linear parabolic (13)-(15). By (8), (11) and the parabolic Schauder theory (for example, see [20]) there exists a unique solution $\bar{v}$, and

$$
\|\bar{v}\|_{C^{2+\sigma, 1+\sigma / 2}\left(\Omega_{T}\right)} \leq A_{0}\|\bar{v}(x, 0)\|_{C^{2+\sigma}(\Omega)} \leq A_{0} M
$$

Similarly, from $U \in X_{M}$, (11) and the parabolic Schauder theory, problem (16)-(18) has a unique solution $\bar{u}$ satisfying

$$
\begin{aligned}
\|\bar{u}\|_{C^{2+\sigma, 1+\sigma / 2}\left(\Omega_{T}\right)} & \leq A_{0}\left(\|\bar{u}(x, 0)\|_{C^{2+\sigma}(\Omega)}+\|\alpha C\|_{C^{\sigma, \sigma / 2}\left(\Omega_{T}\right)}\right) \\
& \leq A_{0} M
\end{aligned}
$$

We now turn to the linear parabolic problem (19)-(21). Using $U \in X_{M}$, (23) and (24) and noting $V_{1}^{\prime}(c)$ and 
$V_{2}^{\prime}(c)$ are Lipschitz continuous, we have

$$
\left\|h_{1}\right\|_{C^{\sigma, \sigma / 2}\left(\Omega_{T}\right)} \leq A_{0} M,
$$

Hence, by Schauder theory as before, the problem (19)(21) admits a unique solution $\bar{C}$ satisfying

$$
\begin{aligned}
& \|\bar{c}\|_{C^{2+\sigma, 1+\sigma / 2}\left(\Omega_{T}\right)} \\
\leq & A_{0}\left(\|\bar{c}(x, 0)\|_{C^{2+\sigma}(\Omega)}+\left\|h_{1}\right\|_{C^{\sigma, \sigma / 2}\left(\Omega_{T}\right)}\right) \leq A_{0} M
\end{aligned}
$$

We conclude from (23), (24) and (26) that

$$
\|\bar{U}\|_{C^{2+\sigma, 1+\sigma / 2}\left(\Omega_{T}\right)} \leq A_{0} M .
$$

By direct calculations, we obtain

$$
\begin{aligned}
& \|\bar{U}(x, t)-\bar{U}(x, 0)\|_{C^{1+\sigma, \sigma / 2}\left(\Omega_{T}\right)} \\
& \leq A_{0} \eta(T)\|\bar{U}\|_{C^{1+\sigma, \sigma / 2}\left(\Omega_{T}\right)}
\end{aligned}
$$

where $\eta(T) \rightarrow 0$ if $T \rightarrow 0$. If we further take $T$ sufficiently small, then by (27)

$$
\begin{aligned}
& \|\bar{U}(x, t)\|_{C^{1+\sigma, \sigma / 2}\left(\Omega_{T}\right)} \leq\|\bar{U}(x, 0)\|_{C^{1+\sigma}(\Omega)} \\
& +\|\bar{U}(x, t)-\bar{U}(x, 0)\|_{C^{1+\sigma, \sigma / 2}\left(\Omega_{T}\right)} \\
\leq & \|\bar{U}(x, 0)\|_{C^{1+\sigma}(\Omega)}+A_{0} \eta(T)\|\bar{U}\|_{C^{2+\sigma, 1+\sigma / 2}\left(\Omega_{T}\right)} \\
\leq & \|\bar{U}(x, 0)\|_{C^{1+\sigma}(\Omega)}+A_{0} \eta(T) M \\
\leq & \|\bar{U}(x, 0)\|_{C^{1+\sigma}(\Omega)}+1 \\
\leq & M .
\end{aligned}
$$

Hence, $\bar{U} \in X_{M}$, i.e. $F$ maps $X_{M}$ into itself. We next show that $F$ is a contraction mapping. Take $U_{1}, U_{2}$ in $X_{M}$, and set $\bar{U}_{1}=F U_{1}$ and $\bar{U}_{2}=F U_{2}$. setting

$$
\delta=\left\|U_{1}-U_{2}\right\|
$$

We derive from (13) that

$$
\begin{aligned}
\delta= & \left\|U_{1}-U_{2}\right\| \frac{\partial\left(\bar{v}_{1}-\bar{v}_{2}\right)}{\partial t}-D_{v} \Delta\left(\bar{v}_{1}-\bar{v}_{2}\right) \\
& +F\left(u_{1}\right)\left(\bar{v}_{1}-\bar{v}_{2}\right)=\bar{v}_{2}\left(F\left(u_{2}\right)-F\left(u_{1}\right)\right),
\end{aligned}
$$

where

$$
\begin{aligned}
& \left\|\bar{v}_{2}\left(F\left(u_{2}\right)-F\left(u_{1}\right)\right)\right\|_{C^{\sigma, \sigma / 2}\left(\Omega_{T}\right)} \\
& \leq A_{0}\left\|U_{1}-U_{2}\right\|_{C^{\sigma, \sigma / 2}\left(\Omega_{T}\right)} \\
& \leq A_{0} \delta .
\end{aligned}
$$

Hence, since $\left.\left(\bar{v}_{1}-\bar{v}_{2}\right)\right|_{t=0}=0$, Schauder theory yields

$$
\left\|\bar{v}_{1}-\bar{v}_{2}\right\|_{C^{2+\sigma, 1+\sigma / 2}\left(\Omega_{T}\right)} \leq A_{0} \delta .
$$

Similarly, we derive from (16) and $\left.\left(\bar{u}_{1}-\bar{u}_{2}\right)\right|_{t=0}=0$, that

$$
\begin{aligned}
\left\|\bar{u}_{1}-\bar{u}_{2}\right\|_{C^{2+\sigma, 1+\sigma / 2}\left(\Omega_{T}\right)} & \leq A_{0}\left\|C_{1}-C_{2}\right\|_{C^{\sigma, \sigma / 2}\left(\Omega_{T}\right)} \\
& \leq A_{0}\left\|U_{1}-U_{2}\right\|_{C^{\sigma, \sigma / 2}\left(\Omega_{T}\right)} \\
& \leq A_{0} \delta
\end{aligned}
$$

We next turn to the equation for $\bar{c}_{1}-\bar{c}_{2}$ :

$$
\frac{\partial\left(\bar{c}_{1}-\bar{c}_{2}\right)}{\partial t}-D_{c} \Delta\left(\bar{c}_{1}-\bar{c}_{2}\right)=h_{2}
$$

where

$$
\begin{aligned}
h_{2}= & -\chi V_{1}\left(c_{1}\right) \Delta\left(\bar{u}_{1}-\bar{u}_{2}\right)-\chi \Delta \bar{u}_{2}\left(V_{1}\left(c_{1}\right)-V_{1}\left(c_{2}\right)\right) \\
& -\xi V_{2}\left(c_{1}\right) \Delta\left(\bar{v}_{1}-\bar{v}_{2}\right)-\xi \Delta \bar{v}_{2}\left(V_{2}\left(c_{1}\right)-V_{2}\left(c_{2}\right)\right) \\
& -\chi V_{1}^{\prime}\left(c_{1}\right) \nabla c_{1} \cdot \nabla\left(\bar{u}_{1}-\bar{u}_{2}\right)-\chi V_{1}^{\prime}\left(c_{1}\right) \nabla \bar{u}_{2} \cdot \nabla\left(c_{1}-c_{2}\right) \\
& -\chi\left(V_{1}^{\prime}\left(c_{1}\right)-V_{1}^{\prime}\left(c_{2}\right)\right) \nabla \bar{u}_{2} \cdot \nabla c_{2} \\
& -\xi V_{2}^{\prime}\left(c_{1}\right) \nabla c_{1} \cdot \nabla\left(\bar{v}_{1}-\bar{v}_{2}\right)-\xi V_{2}^{\prime}\left(c_{1}\right) \nabla \bar{v}_{2} \cdot \nabla\left(c_{1}-c_{2}\right) \\
& -\xi\left(V_{2}^{\prime}\left(c_{1}\right)-V_{2}^{\prime}\left(c_{2}\right)\right) \nabla \bar{v}_{2} \cdot \nabla c_{2}
\end{aligned}
$$

Noting $V_{1}^{\prime}(c)$ and $V_{2}^{\prime}(c)$ are Lipschitz continuous and using (6), (7), (27), (28) and (29), we have

$$
\left\|h_{2}\right\|_{C^{\sigma, \sigma / 2}\left(\Omega_{T}\right)} \leq A_{0} \delta .
$$

By Schauder theory, since $\left.\left(\bar{c}_{1}-\bar{c}_{2}\right)\right|_{t=0}=0$,

$$
\left\|\bar{c}_{1}-\bar{c}_{2}\right\|_{C^{2+\sigma, 1+\sigma / 2}\left(\Omega_{T}\right)} \leq A_{0}\left\|h_{2}\right\|_{C^{\sigma, \sigma / 2}\left(\Omega_{T}\right)} \leq A_{0} \delta .
$$

Combining this with (28) and (29), we get

$$
\left\|\bar{U}_{1}-\bar{U}_{2}\right\|_{C^{2+\sigma, 1+\sigma / 2}\left(\Omega_{T}\right)} \leq A_{0} \delta .
$$

Nothing $\left(\bar{U}_{1}-\bar{U}_{2}\right)(x, 0) \equiv 0$ and proceeding as before, we have

$$
\begin{aligned}
& \left\|\bar{U}_{1}-\bar{U}_{2}\right\|_{C^{1+\sigma, \sigma / 2}\left(\Omega_{T}\right)} \leq A_{0} \eta(T)\left\|\bar{U}_{1}-\bar{U}_{2}\right\|_{C^{2+\sigma, 1+\sigma / 2}\left(\Omega_{T}\right)} \\
& \leq A_{0} \eta(T) \delta=A_{0} \eta(T)\left\|U_{1}-U_{2}\right\|_{C^{1+\sigma, \sigma / 2}\left(\Omega_{T}\right)}
\end{aligned}
$$

Taking $T$ small such that $A_{0} \eta(T)<\frac{1}{2}$ we conclude from (32) that $F$ is a contraction in $X_{M}$. By the contraction mapping theorem $F$ has a unique fixed point $U$ in $X_{M}$ which is the unique solution of (1)-(5).

\section{A Priori Estimates and Global Existence}

To continue the local solution established in the above section to all $t>0$ we need to establish some a priori estimates. Throughout this section, in addition to the assumptions (6)-(8) and (11) we assume that the assumptions (9) and (10) hold.

Noting $V_{1}(0)=V_{2}(0)=0, c_{0}(x) \geq 0, v_{0}(x) \geq 0$ and $u_{0}(x) \geq 0$, and using the maximum principle, we easily prove the following lemma.

Lemma 4.1. Assume that $U=(c, v, u) \in C^{2,1}\left(\Omega_{T}\right)$ is a solution to (1)-(5), then 


$$
c \geq 0, v \geq 0, u \geq 0 .
$$

Lemma 4.2. Assume that $U=(c, v, u) \in C^{2,1}\left(\Omega_{T}\right)$ is a solution to (1)-(5) then for $p>2$, we have

$$
\begin{aligned}
& \|c\|_{L^{p}\left(\Omega_{T}\right)} \leq A, \\
& \|v\|_{W_{p}^{2,1}\left(\Omega_{T}\right)} \leq A_{0}, \\
& \|u\|_{W_{p}^{2,1}\left(\Omega_{T}\right)} \leq A .
\end{aligned}
$$

Proof. For $p>2$, we derive from

$$
\begin{aligned}
\frac{\mathrm{d}}{\mathrm{d} t} \int_{\Omega} c^{p} \mathrm{~d} x= & p \int_{\Omega} c^{p-1} c_{t} \mathrm{~d} x=-p(p-1) D_{c} \int_{\Omega} c^{p-2}|\nabla c|^{2} \mathrm{~d} x \\
& +\chi p(p-1) \int_{\Omega} c^{p-2} V_{1}(c) \nabla c \cdot \nabla u \mathrm{~d} x \\
& +\xi p(p-1) \int_{\Omega} c^{p-2} V_{2}(c) \nabla c \cdot \nabla v \mathrm{~d} x \\
\leq & \frac{-4(p-1) D_{c}}{p} \int_{\Omega}\left|\nabla c^{2 / 2}\right|^{2} \mathrm{~d} x \\
& +A_{0} \chi p(p-1) \int_{\Omega} c^{p-2} \nabla c \cdot \nabla u \mathrm{~d} x \\
& +\xi p(p-1) \int_{\Omega} c^{p-2} V_{2}(c) \nabla c \cdot \nabla v \mathrm{~d} x .
\end{aligned}
$$

We now consider the integral $\int_{\Omega} c^{p-2} \nabla c \cdot v \nabla u \mathrm{~d} x$. By Equation (3) and the parabolic $L^{p}$ estimate [20] we have

$$
\begin{aligned}
\|u\|_{W_{p}^{2,1}\left(\Omega_{T}\right)} & \leq A_{0}\left(\left\|u_{0}\right\|_{W_{p}^{2}(\Omega)}+\|\alpha C\|_{L^{p}\left(\Omega_{T}\right)}\right) \\
& \leq A_{0}+A_{0}\|c\|_{L^{p}\left(\Omega_{T}\right)},
\end{aligned}
$$

In particular,

$$
\|u\|_{L^{p}\left(\Omega_{T}\right)}+\left\|u_{t}\right\|_{L^{p}\left(\Omega_{T}\right)} \leq A_{0}+A_{0}\|c\|_{L^{p}\left(\Omega_{T}\right)}
$$

Multiplying Equation (3) by $c^{p-1}$ and integrating in $\Omega_{t}$, we obtain

$$
\begin{gathered}
(p-1) D_{u} \int_{0}^{t} \int_{\Omega} c^{p-2} \nabla c \cdot \nabla u \mathrm{~d} x \mathrm{~d} t=\alpha \int_{0}^{t} \int_{\Omega} c^{p} \mathrm{~d} x \mathrm{~d} t \\
-\beta \int_{0}^{t} \int_{\Omega}^{p-1} c^{p-1} u \mathrm{~d} x \mathrm{~d} t-\int_{0}^{t} \int_{\Omega} c^{p-1} u_{t} \mathrm{~d} x \mathrm{~d} t
\end{gathered}
$$

And there for, by Young's inequality and estimate (39), we have

$$
\begin{aligned}
& (p-1) D_{u} \int_{0}^{t} \int_{\Omega} c^{p-2} \nabla c \cdot \nabla u \mathrm{~d} x \mathrm{~d} t \leq A_{0}(p) \int_{0}^{t} \int_{\Omega} c^{p} \mathrm{~d} x \mathrm{~d} t \\
& +\int_{0}^{t} \int_{\Omega}|u|^{p} \mathrm{~d} x \mathrm{~d} t+\int_{0}^{t} \int_{\Omega}\left|u_{t}\right|^{p} \mathrm{~d} x \mathrm{~d} t \leq A_{0}(p) \int_{0}^{t} \int_{\Omega} c^{p} \mathrm{~d} x \mathrm{~d} t+A_{0} .
\end{aligned}
$$

Also, by Equation (2) and the parabolic $L^{p}$ estimate we have

$$
\|v\|_{W_{p}^{2,1}\left(\Omega_{T}\right)} \leq A_{0}\left\|v_{0}\right\|_{W_{p}^{2}(\Omega)} \leq A_{0}
$$

In particular,

$$
\|\nabla v\|_{L^{p}\left(\Omega_{T}\right)} \leq A_{0} .
$$

By (9), Young's inequality and estimate (42)

$$
\begin{aligned}
& \int_{0}^{t} \int_{\Omega} c^{p-2} V_{2}(\mathrm{c}) \nabla c \cdot \nabla v \mathrm{~d} x \mathrm{~d} t \leq \mathrm{A}_{0} \int_{0}^{t} \int_{\Omega} c^{p-2}|\nabla c||\nabla v| \mathrm{d} x \mathrm{~d} t \\
& \leq \epsilon \int_{0}^{t} \int_{\Omega}^{p-2} c^{p-2}|\nabla c|^{2} \mathrm{~d} x \mathrm{~d} t+A_{0}(\epsilon) \int_{0}^{t} \int_{\Omega} c^{p-2}|\nabla v|^{2} \mathrm{~d} x \mathrm{~d} t \\
& \leq \frac{4 \epsilon}{p^{2}} \int_{0}^{t} \int_{\Omega}\left|\nabla c^{p / 2}\right|^{2} \mathrm{~d} x \mathrm{~d} t+A_{0}(p, \epsilon) \iint_{0}^{t} \int_{\Omega}^{p} c^{p} \mathrm{~d} x \mathrm{~d} t \\
& \quad+\int_{0}^{t} \int_{\Omega}|\nabla v|^{p} \mathrm{~d} x \mathrm{~d} t \leq \frac{4 \epsilon}{p^{2}} \int_{0}^{t} \int\left|\nabla c^{p / 2}\right|^{2} \mathrm{~d} x \mathrm{~d} t \\
& \quad+A_{0}(p, \epsilon) \int_{0}^{t} \int_{\Omega} c^{p} \mathrm{~d} x \mathrm{~d} t+A_{0}
\end{aligned}
$$

Integrating with respect to $t$ on both sides (37), noting (11) and using estimates (40) and (43) and taking $\epsilon$ sufficiently small, we obtain

$$
\begin{aligned}
\int_{\Omega} c^{p} \mathrm{~d} x \leq & \frac{4(p-1)}{p}\left(\xi \epsilon-D_{c}\right) \int_{0}^{t} \int_{\Omega}\left|\nabla c^{p / 2}\right|^{2} \mathrm{~d} x \mathrm{~d} s \\
& +A_{0}(p, \epsilon) \int_{0}^{t} \int_{\Omega} c^{p}+A_{0}(p) \\
\leq & A_{0}(p) \int_{0}^{t} \int_{\Omega} c^{p} \mathrm{~d} x \mathrm{~d} t+A_{0}(p) .
\end{aligned}
$$

Gronwall's lemma yields

$$
\int_{0}^{T} \int_{\Omega} c^{p} \mathrm{~d} x \mathrm{~d} t \leq A(p) .
$$

Now, by (39) and (44) we have

$$
\|u\|_{W_{p}^{2,1}\left(\Omega_{T}\right)} \leq A(p) .
$$

This completes the proof of lemma 4.2.

In the following result we obtaina better bound of $c, a$ $L^{\infty}$-bound. Let $p>1$ and define $B:=-D_{c} \Delta+I$, with domain $D(B)=\left\{c \in W_{p}^{2}(\Omega): \frac{\partial c}{\partial n}=0\right.$ on $\left.\partial \Omega\right\}$ For each $\theta \geq 0$, define the sectorial operator $B^{\theta}$ (see [21]) and $X_{\theta}:=D\left(B^{\theta}\right)$ with the norm $\|c\|_{X_{\theta}}=\left\|B^{\theta} c\right\|_{L^{p}}$.

Lemma 4.3. Let $2 \theta<1$, then for $t \in\left[t_{0}, T\right)$ where $t_{0}>0$, we have

$$
\|c(t)\|_{X_{\theta}} \leq A .
$$

Proof. We have that

$$
\begin{aligned}
& c(t)=e^{-t B} c_{0} \\
& +\int_{0}^{t} e^{-(t-s) B}\left[-\nabla \cdot\left(\chi V_{1}(c) \nabla u\right)-\nabla \cdot\left(\xi V_{2}(c) \nabla v\right)+c\right] \mathrm{d} s
\end{aligned}
$$

and so 


$$
\begin{aligned}
& \|c(t)\|_{X_{\theta}} \leq\|\| e^{-t B} C_{0} \|_{X_{\theta}} \\
& +\int_{0}^{t}\left\|e^{-(t-s) B}\left[-\nabla \cdot\left(\chi V_{1}(c) \nabla u\right)-\nabla \cdot\left(\xi V_{2}(c) \nabla v\right)+c\right]\right\|_{X_{\theta}} \mathrm{d} s
\end{aligned}
$$

By [21] (Theorem 1.4.3) and (11)

$$
\left\|e^{-t B} c_{0}\right\|_{X_{\theta}} \leq A_{0} t^{-\theta} e^{-\varrho t}\left\|c_{0}\right\|_{L^{p}(\Omega)} \leq A_{0} t^{-\theta} e^{-\varrho t}
$$

and, by (34),

$$
\begin{aligned}
\left\|e^{-(t-s) B} C\right\|_{X_{\theta}} & \leq A_{0}(t-s)^{-\theta} e^{-\varrho(t-s)}\|C\|_{L^{p}(\Omega)} \\
& \leq A(t-s)^{-\theta} e^{-\varrho(t-s)},
\end{aligned}
$$

where $Q \in(0,1)$. Moreover, by [22] (Lemma 2.1), (9), (35) and (36), we obtain

$$
\begin{aligned}
& \left\|e^{-(t-s) B}\left[-\nabla \cdot\left(\chi V_{1}(c) \nabla u\right)-\nabla \cdot\left(\xi V_{2}(c) \nabla v\right)\right]\right\|_{X_{\theta}} \\
& \leq A_{0}\left\|e^{(t-s) \Delta}\left[-\nabla \cdot\left(\chi V_{1}(c) \nabla u\right)-\nabla \cdot\left(\xi V_{2}(c) \nabla v\right)\right]\right\|_{X_{\theta}} \\
& \leq A_{0}(\epsilon)(t-s)^{-\theta-\frac{1}{2}-\epsilon} e^{-\rho(t-s)}\left\|\chi V_{1}(c) \nabla u+\xi V_{2}(c) \nabla v\right\|_{L^{p}(\Omega)} \\
& \leq A(\epsilon)(t-s)^{-\theta-\frac{1}{2}-\epsilon} e^{-\rho(t-s)}
\end{aligned}
$$

where $\epsilon>0$ such that $-\theta-\frac{1}{2}-\epsilon>-1$.

Inserting (48)-(50) into (47) and noting $\theta+\frac{1}{2}+\epsilon<1$ and $\theta<1$, we obtain

$$
\begin{aligned}
& \|c(t)\|_{X_{\theta}} \leq A_{0} t^{-\theta} e^{-\rho t} \\
& +A(\epsilon) \int_{0}^{t}\left[(t-s)^{-\theta-\frac{1}{2}-\epsilon} e^{-\varrho(t-s)}+(t-s)^{-\theta} e^{-\rho(t-s)}\right] \mathrm{d} s \\
& \leq A\left(t_{0}\right)
\end{aligned}
$$

for all $t \in\left[t_{0}, T\right)\left(0<t_{0}<T\right)$.

This completes the proof of lemma 4.3.

Lemma 4.4. We have that

$$
\|c(t)\|_{L^{\infty}(\Omega)} \leq A \text { for all } t \in[0, T) .
$$

Proof. Let $P>d, 2 \theta \in\left(\frac{d}{p}, 1\right)$. since $2 \theta>\frac{d}{p}$ we have by [21] (Theorem 1.6.1) that

$$
X_{\theta} \rightarrow c(\bar{\Omega})
$$

Thanks to lemma 4.3 we have that

$$
\|c(t)\|_{L^{\infty}(\Omega)} \leq A\left(t_{0}\right) \text { for } t>t_{0}>0 .
$$

Moreover, the local existence Theorem yields $\|c(t)\|_{L^{\infty}(\Omega)} \leq A_{0}$ for $t<t_{0}$.
Therefore

$$
\|c(t)\|_{L^{\infty}(\Omega)} \leq A \text { for all } t \in[0, T) .
$$

This completes the proof of Lemma 4.4.

Lemma 4.5. We have that

$$
\|C\|_{W_{p}^{2,1}\left(\Omega_{T}\right)} \leq A
$$

Proof. By the Sobolev embedding theorem (see [20], (Lemma 3.3, p. 80)), if we take $p$ sufficiently large, then (35) and (36) yields

$$
\|\nabla v\|_{C^{\sigma, \sigma / 2}\left(\Omega_{T}\right)} \leq A_{0,}\|\nabla u\|_{C^{\sigma, \sigma / 2}\left(\Omega_{T}\right)} \leq A,
$$

and therefore

$$
\|\nabla v\|_{L^{\infty}\left(\Omega_{T}\right)} \leq A_{0,}\|\nabla u\|_{L^{\infty}\left(\Omega_{T}\right)} \leq A,
$$

By (7) and (51), we have

$$
\left\|V_{1}^{\prime}(c)\right\|_{L^{\infty}(\Omega)} \leq A,\left\|V_{2}^{\prime}(c)\right\|_{L^{\infty}(\Omega)} \leq A .
$$

Now, Equation (1) can be rewritten as

$$
\begin{aligned}
& \frac{\partial c}{\partial t}-D_{C} \Delta c+\left(\chi V_{1}^{\prime}(c) \nabla u+\xi V_{2}^{\prime}(c) \nabla v\right) \cdot \nabla c \\
& =-\chi V_{1}(c) \Delta u-\xi V_{2}(c) \Delta v
\end{aligned}
$$

where

$$
\begin{gathered}
\left\|\chi V_{1}^{\prime}(c) \nabla u+\xi V_{2}^{\prime}(c) \nabla v\right\|_{L^{\infty}(\Omega)} \leq A \\
\left\|\chi V_{1}(c) \Delta u+\xi V_{2}(c) \Delta v\right\|_{L^{p}\left(\Omega_{T}\right)} \leq A
\end{gathered}
$$

By (9), (35), (36), (53) and (55). These, along with (11) and the parabolic $L^{p}$ estimate, yield the estimate (52).

Lemma 4.6. Assume that $U=(c, v, u) \in C^{2,1}\left(\Omega_{T}\right)$ is a solution to (1)-(5), then

$$
\|U\|_{C^{2+\sigma, 1+\sigma / 2}\left(\Omega_{T}\right)} \leq A \text {. }
$$

Proof. By (52) and the Sobolev embedding theorem (taking $p$ large),

$$
\|c\|_{C^{\sigma, \sigma / 2}\left(\Omega_{T}\right)} \leq A
$$

Also, (35), (36) and the Sobolev embedding theorem (taking $p$ large) yield

$$
\|v\|_{C^{\sigma, \sigma / 2}\left(\Omega_{T}\right)} \leq A_{0},\|u\|_{C^{\sigma, \sigma / 2}\left(\Omega_{T}\right)} \leq A .
$$

Now, from (3), (11), (59) and the parabolic Schauder estimates we have

$$
\|u\|_{C^{2+\sigma, 1+\sigma / 2}\left(\Omega_{T}\right)} \leq A
$$

Also, the parabolic Schauder estimates yield

$$
\|v\|_{C^{2+\sigma, 1+\sigma / 2}\left(\Omega_{T}\right)} \leq A_{0} .
$$

Finally, we conclude from (61) and (62) that 


$$
\begin{aligned}
& \left\|\chi V_{1}^{\prime}(c) \nabla u+\xi V_{2}^{\prime}(c) \nabla v\right\|_{C^{\sigma, \sigma / 2}\left(\Omega_{T}\right)} \leq A \\
& \left\|\chi V_{1}(c) \Delta u+\xi V_{2}(c) \Delta v\right\|_{C^{\sigma, \sigma / 2}\left(\Omega_{T}\right)} \leq A
\end{aligned}
$$

Hence, by the parabolic Schauder estimates, we obtain

$$
\|C\|_{C^{2+\sigma, 1+\sigma / 2}\left(\Omega_{T}\right)} \leq A \text {. }
$$

This completes the proof of Lemma 4.6.

With a priori estimate (58), we can extend the local classical solution established in Theorem 3.1 to all $t>0$, as done in [15]. Namely we have

Theorem 4.7. There exists a unique global solution $U \in C^{2+\sigma, 1+\sigma / 2}\left(\Omega_{T}\right)$ of the system (1)-(5) for any given $T>0$.

\section{REFERENCES}

[1] M. A. J. Chaplain and G. Lolas, "Mathematical Modeling of Cancer Invasion of Tissue: Dynamic Heterogeneity," Network and Heterogeneous Media, Vol. 1, No. 3, 2006, pp. 399-439. doi:10.3934/nhm.2006.1.399

[2] N. Bellomo, N. K. Li and P. K. Maini, "On the Foundation of Cancer Modeling: Selected Topics Speculations, and Perspectives," Mathematical Model and Methods in Applied Sciences, Vol. 18, No. 4, 2008, pp. 593-646. doi:10.1142/S0218202508002796

[3] R. A. Gatenby and E. T. Gawlinski, "A Reaction Diffusion Model of Cancer Invasion," Cancer Research, Vol. 56, No. 24, 1996, pp. 5745-5753.

[4] A. J. Perumpanani and H. M. Byrne, "Extra Cellular Matrix Concentration Exerts Selection Pressure on Invasive Cells," European Journal of Cancer, Vol. 35, No. 8, 1999, pp. 1274-1280. doi:10.1016/S0959-8049(99)00125-2

[5] A. Gerisch and M. A. J. Chaplain, "Mathematical Modeling of Cancer Cell Invasion of Tissue: Local and NonLocal Models and the Effect Adhesion," Journal of Theoretical Biology, Vol. 250, No. 4, 2008, pp. 684-704. doi:10.1016/i.jtbi.2007.10.026

[6] Z. Szymańsska, C. Morales-Rodrigo, M. Lachowiz and M. Chaplain, "Mathematical Modelling of Cancer Invasion of Tissue: The Role and Effect of Non-Local Interactions," Mathematical Model and Methods in Applied Sciences, Vol. 19, No. 2, 2009, pp. 257-281.

[7] Z. Szymańsska, J. Urbański and A. Marciniak-Czochra, "Mathematical Modeling of the Influence Heat Shock Proteins on Cancer Invasion of Tissue," Journal of Mathematical Biology, Vol. 58, No. 4-5, 2009, pp. 819-844. doi:10.1007/s00285-008-0220-0

[8] C. Walker and G. F. Webb, "Global Existence of Classical Solutions for a Haptotaxis Model," SIAM Journal on Mathematical Analysis, Vol. 38, No. 5, 2007, pp. 16941713. doi: $10.1137 / 060655122$

[9] M. A. J. Chaplain and A. R. A. Anderson, "Mathematical Modeling of Tissue Invasion," Chapman \& Hall/CRT,
London/Boca Raton, 2003, pp. 267-297.

[10] C. Walker, "Global Existence for an Age and Spatially Structured Haptotaxis Model with Nonlinear Age-Boundary Conditions," European Journal of Applied Mathematics, Vol. 19, No. 2, 2008, pp. 113-147. doi:10.1137/060655122

[11] A. Marciniak-Czochra and M. Ptashnyk, "Boundedness of Solutions of a Haptotaxis Modelsmodel," Mathematical Model and Methods in Applied Sciences, Vol. 20, No. 3, 2010, pp. 449-476. doi:10.1142/S0218202510004301

[12] G. Litcanu and C. Morales-Rodrigo," Global Solutions and Asymptotic Behavior for a Parabolic Degenerate Coupled System Arising from Biology," Nonlinear Analysis, Vol. 72, No. 1, 2010, pp .77-98.

[13] G. Litcanu and C. Morales-Rodrigo, "Asymptotic Behavior of Global Solutions to a Model of Cell Invasion," Mathematical Model and Methods in Applied Sciences, Vol. 20, No. 9, 2009, pp. 1721-1758.

[14] M. Lachowiz, "Towards Microscopic and Non-Local Models of Tumor Invasion of Tissue," In: N. Bellomo, M. Chaplain and E. De Angelis, Eds., Selected Topics in Cancer Modeling, Birkhäuser, Boston, 2008, pp. 49-63. doi:10.1007/978-0-8176-4713-1 3

[15] Y. Tao and M. Wang, "Global Solution for a Chemotactic-Haptotactic Model of Cancer Invasion," Nonlinearity, Vol. 21, No. 10, 2008, pp. 2221-2238. doi:10.1088/0951-7715/21/10/002

[16] Y. Tao, "Global Existence of Classical Solutions to a Combined Chemotaxis-Haptotaxis Model with Logistic Source," Journal of Mathematical Analysis and Applications, Vol. 354, No. 1, 2009, pp. 60-69. doi:10.1016/j.jmaa.2008.12.039

[17] Y. Tao and M. Wang, "A Combined Chemotaxis-Haptotaxis System: The Role of Logistic Source," IAM Journal on Mathematical Analysis, Vol. 41, No. 4, 2009, pp. 1533 1558. doi:10.1137/090751542

[18] Y. Tao and C. Cui, "A Density-Dependent ChemotaxisHaptotaxis System Modeling Cancer Invasion," Journal of Mathematical Analysis and Applications, Vol. 367, No. 2, 2010, pp. 612-624. doi:10.1016/i.jmaa.2010.02.015

[19] B. E. Ainseba, M. Bendahman and A. Noussair, "A Reaction-Diffusion System Modeling Predator-Prey with PreTaxis," Nonlinear Analysis: Real Word Application, Vol. 9, No. 5, 2008, pp. 2086-2105. doi:10.1016/j.nonrwa.2007.06.017

[20] O. A. Ladyzenskaja, V. A. Solonnikov and N. N. Uarl'ceva, "Linear and Quasi-Linear Equations of Parabolic Type," American Mathematical Society, Providence, 1968.

[21] D. Henry, "Geometric Theory of Semilinear Parabolic Equations," In: Lecture Notes Mathematics, Vol. 840, Springer, Berlin, 1981.

[22] D. Horstmann and M. Winkler, "Bound Endless vs. BlowUp in a Chemotaxis System," Journal of Differential Equations, Vol. 215, No. 1, 2005, pp. 52-107. doi:10.1016/j.jde.2004.10.022 